\title{
Study on Luminescence Properties of Blue phosphorescent Microcavity Organic Light-emitting Devices \\ Chun-Yu ZHANG ${ }^{1, a}$, Ding-Dong WANG ${ }^{2, b}$, Hua RONG ${ }^{3, c}$ \\ ${ }^{1,2,3}$ School of Materials Science and Engineering, Jilin Jianzhu University, Changchun 130118, China \\ azhangccy68@163.com, b2352143864@qq.com, c27814247@qq.com
}

Keywords: Organic light-emitting device(OLED), Phosphorescent, Microcavity.

\begin{abstract}
A blue phosphorescent cavity organic light-emitting Devices (MOLED) is obtained. The structure is Glass/ DBR/ ITO $/ \mathrm{MoO}_{3}(1 \mathrm{~nm}) / \mathrm{TcTa}(40 \mathrm{~nm}) / \mathrm{CBP}:$ FIRPIC(30nm, 6\%)/ TPBI(35nm)/ $\mathrm{LiF}(1 \mathrm{~nm}) / \mathrm{Al}(80 \mathrm{~nm})$. For comparing, the OLED without cavity is also fabricated. The effect of microcavity structure on phosphorescent luminescent device is discussed. The result is shown that electroluminescence (EL) spectrum peak of OLED is on 470nm, the full width at half maximum (FWHM) is $60 \mathrm{~nm}$ and the electroluminescence (EL) spectrum peak of MOLED is 470nm, the FWHM is $26 \mathrm{~nm}$. That means that the latter is narrowed around the half compared with the former. The luminescence spectrum of OLED with microcavity structure can be narrowed, therefore color purity is improved. For MOLED, the maximum brightness and the maximum external quantum efficiency (EQE) is $9720 \mathrm{~cd} / \mathrm{m}^{2}$ and $16.4 \%$,respectively. For OLED, the maximum brightness and external quantum efficiency is $9020 \mathrm{~cd} / \mathrm{m}^{2}$ and $14.2 \%$, respectively. The experimental results show that the luminescence properties of microcavity devices have been improved greatly compared with the no cavity devices.
\end{abstract}

\section{Introduction}

Organic Light-emitting Devices (OLED) is a new showing technology because many advancement, for example, light weight, thin body, high brightness, fast respond, low voltage and high effect [1]. The potential capability on illumination is get much more attention [2]. The first OLED was reported by Pope on 1963 with anthracene monocrystal [3]. And on 1987, the OLED was made with vacuum evaporation by C.W.Tang and S.A Slyke[4], which performed double layer with hole transport layer and $\mathrm{Alq}_{3}$ as emit layer. Another milestone on OLED was the phosphorescence as emit on 1998. That work was done by Baldo and Forrest, and triplet phosphorescence was performed at room temperature [5]. The limit of the inter quantum effect could be promote from $25 \%$ to $100 \%$ theoretically because the phosphorescence emit could translate $75 \%$ the energy of triplet. Therefore the organic phosphorescence is hoped to be next generation illumination material with high effect, high energy translating and high brightness [6-7]. The main problem now is that concentration quenching and triplet quenching always occur when the proportion of phosphorescence is high. To solute this problem, the phosphorescence is mingled to fundamental material according to a certain proportion, in general.

Optical cavity is a micro resonant cavity with wavelength size. It includes plan microcavity, sphere microcavity and ring microcavity. The fundamental type is the one dimension plan cavity with FP construction. It is composed with a set of plan mirrors. The emit light in resonant model is gotten strength by interfering. The resonant model is the cavity size is the integral multiples or the phase is the integral multiple of $2 \pi[8]$ :

$$
\left(\varphi_{1}+\varphi_{2}\right)-\frac{4 \pi}{\lambda} \sum_{i} n_{i} d_{i} \cos \left[\arcsin \left(\frac{\sin \theta}{n_{i}}\right)\right]=2 m \pi
$$

Where $\varphi_{2}$ and $\varphi_{1}$ are the reflective phase shift of DBR and metal mirror, respectively. $\lambda$ is 
resonant wavelength, $n_{i}$ and $d_{i}$ is the refractive index and thick of layers, respectively. $L$ is the sum of , which is the microcavity size. $\theta$ is the measurement angle of outside. $m$ is the grade of model, which is the integral. Recently, the cavity is applied on OLED to promote the character of luminance and much more results are obtained. [9-10]

A cavity with blue phosphorescence is obtained in our laboratory now. And for compare, an OLED with the same material but without cavity is made of also. The resonant wavelength is $450 \mathrm{~nm}$. It is match the reflective area of DBR reflective mirror. The calculation is made on the FP equation and relevant equations to optimize the result for the thick of layers and keeping the whole length is on the resonant model.

The both results of luminance construction are, respectively:

MOLED: Glass/DBR /ITO / $\mathrm{MoO}_{3}(1 \mathrm{~nm})$ / TcTa (40nm) / CBP: FIRPIC (30nm, 6\%) / TPBI $(35 \mathrm{~nm}) / \mathrm{LiF}(1 \mathrm{~nm}) / \mathrm{Al}(80 \mathrm{~nm})$.

OLED: Glass/ITO / $\mathrm{MoO}_{3}(1 \mathrm{~nm}) / \mathrm{TcTa}(40 \mathrm{~nm}) / \mathrm{CBP}:$ FIRPIC (30nm, 6\%) / TPBI (35nm) /LiF $(1 \mathrm{~nm}) / \mathrm{Al}(80 \mathrm{~nm})$.

The substrate is glass. The luminance source is the blue phosphorescence FIRPIC which is mingle to fundamental material CBP. The positive material is $\mathrm{MoO}_{3}$. The hole injection is TcTa. The translating is TPBI. The negative pole is high reflective $\mathrm{Al}$ and LiF. The positive pole is ITO. The transparent Bragg reflection (DBR) of the MOLED is parallel to Al mirror to construct the cavity. The central wavelength of the cavity bottom DBR is designed to blue $\lambda=450 \mathrm{~nm}$. The period is 2(HL)ITO. The high index of refraction is used as $\mathrm{H}: \mathrm{TiO}_{2}, \mathrm{n}=2.05$; and low index of refraction is as $\mathrm{L}: \mathrm{SiO}_{2}, \mathrm{n}=1.46$; the index of refraction of ITO is 2.0 , the optical thick of every material is the quarter of $\lambda$.The real thick of ITO is $56 \mathrm{~nm}$. The luminance area is $1 \mathrm{~mm} * 1 \mathrm{~mm}$.

\section{Experimental}

The substrate of MOLED DBR is made from both reflective mirror and ITO conducting film as positive pole on the glass fundamental, which is obtained with Electron Beam Coating Machine. The substrate of OLED is made from only ITO conducting film as positive pole on the glass fundamental. The sheet resistance of ITO is $100 \Omega / \square$ on the both cases. The art of clean is follow: the glass fundament is sonicleaned by ethyl alcohol, acetone and demonized water consequently for $5 \mathrm{~min}$. And then it is dying with high pressed nitrogen. After that it is deal with $\mathrm{O}_{2}$ Plasma for $1 \mathrm{~min}$. The organic materials then are coated in the coating machine successively. The luminance materials and the blue phosphorescence are put in different evaporate body respectively. They are used different heating source to keep the evaporate at the same time for mingle. The evaporated velocity is adjusted with the temperature of evaporated body. Therefore the proportion of mingling is adjusted. The last step is coating $\mathrm{LiF}$ and aluminum film in the melt coating machine. The aluminum film is the negative pole as well as another reflective mirror. All of the vacuum is better than $5^{*} 10^{-4} \mathrm{~Pa}$, the rate of deposition is $0.1 \sim 0.2 \mathrm{~nm} / \mathrm{s}$. When element is set, the substrate is heated up to $60^{\circ} \mathrm{C}$. The thickness of films and the rates of growing are adjusted by quartz crystal oscillator, which is calibrated by step profiler. The element is measured on the room condition without sealed when it is finished. The characteristics of electric and spectral are measured with the laboratory measurement system which is constructed by driving power 2611 and PR705 scan photometer.

\section{Result and Discussion}

For compare the physical characters, the thickness and orders of each layer are the same for both elements. The different, only, is with or without mirror. That means one element (MOLED) is with cavity and another one (OLED) is without the cavity.

Fig. 1 shows the reflective spectrum just on the up position of the MOLED substrate with DBR. The maximum of the reflective ratio is $57.1 \%$ which is on the centric wavelength position. The designed area of high reflective ration is limited a certain width near the blue wavelength. This point is the advantages of medium cavity than the melt cavity. That means cavity could enforce the 
blue effective so that the pure of color could be promoted and so on.

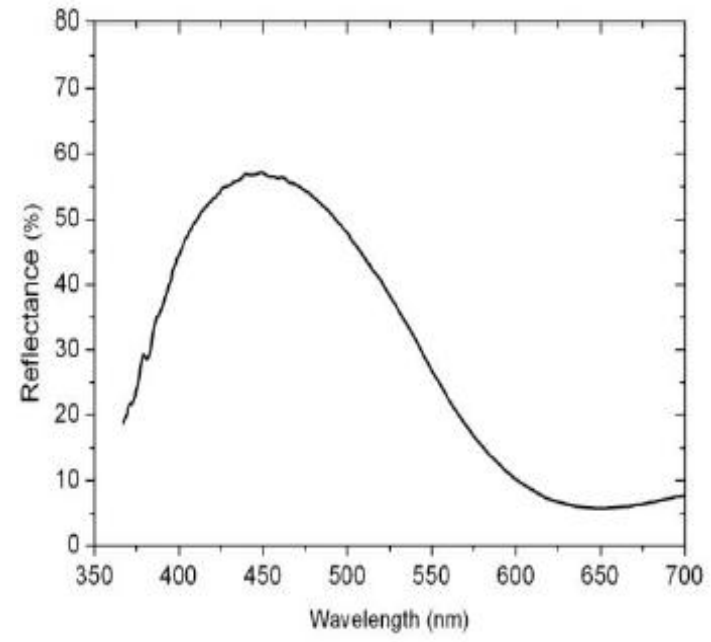

Fig.1 Reflectance spectrum of DBR

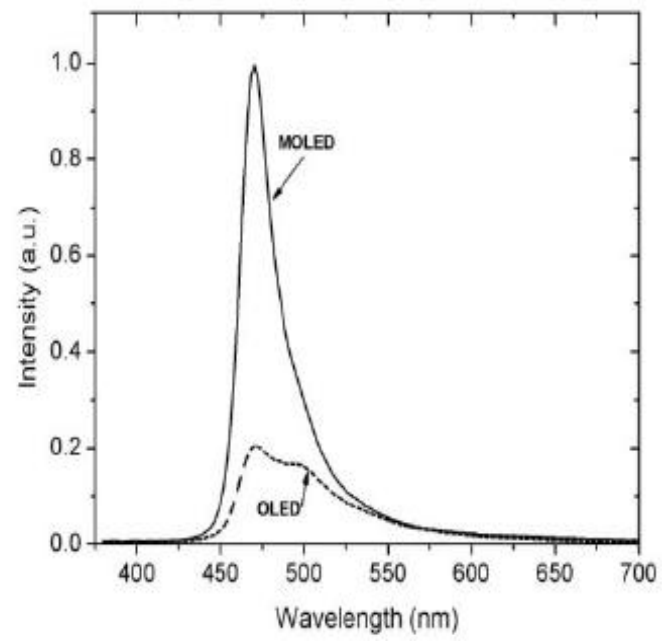

Fig.2 EL spectrum of the MOLED and OLED

Fig.2 shows the comparative of spectrum of EL between the MOLED and OLED. The full line represents the electroluminescence (EL) of MOLED and imaginary line represents the electroluminescence (EL) of OLED. It is clearly that the peak of the OLED is 470mm, and FWHM is $60 \mathrm{~nm}$, and there is another shoulder peak on $496 \mathrm{~nm}$. There is only one peak of MOLED is on $470 \mathrm{~nm}$, and its FWHM is $26 \mathrm{~nm}$. The FWHM of element with cavity is narrow up to $43 \%$, and the strength of the peak is up to 4.8 fold. This is shown the pure of the spectrum is promoted with the cavity construction.

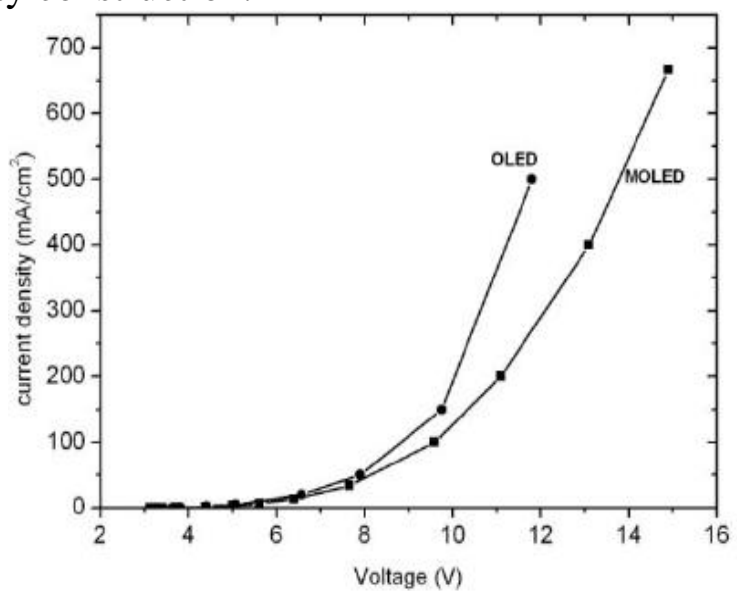

Fig.3 Currert density-Voltage characteristics of OLED and MOLED

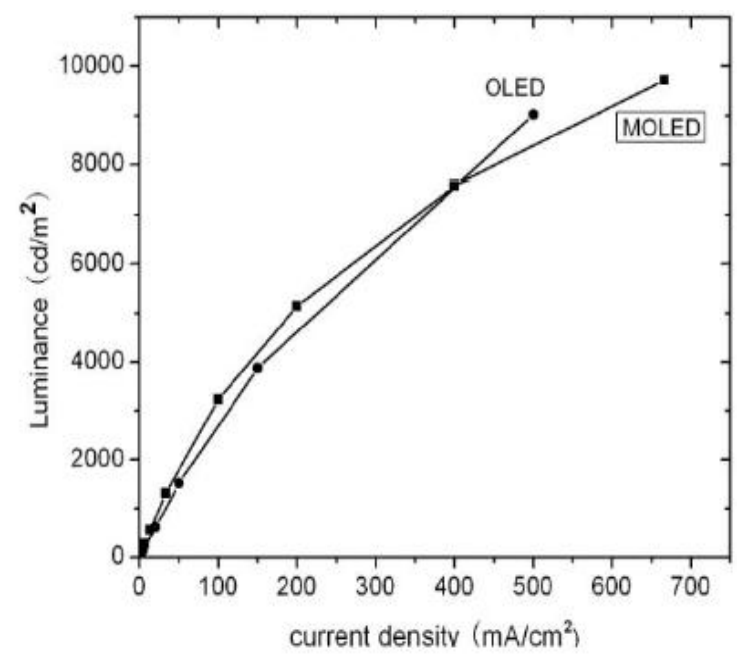

Fig.4 Luminance-Current density characteristics of the MOLED and OLED

Fig.3 shows the current density-voltage characteristics of MOLED and OLED. The solid square points represent the data of MOLED. The sold points represent the data of OLED(the same meaning in the Fig.4 and Fig.5). The start voltage is 3.15V for OLED, and 3.12V for MOLED. The current density of both OLED and MOLED are increased with the voltage. That is shown clearly that both MOLED and OLED have perfect rectification characteristic of diode.

Fig.4 shows the luminance-current density characteristics of the MOLED and OLED. It is clearly that the luminance is strengthened with the current density. The luminance of element with the cavity is great than that without cavity when the current density is below the $400 \mathrm{~mA} / \mathrm{cm}^{2}$. And it is contrast when the current density is up the $400 \mathrm{~mA} / \mathrm{cm}^{2}$. The maximum of luminance are $9720 \mathrm{~cd} / \mathrm{m}^{2}$ and $9020 \mathrm{~cd} / \mathrm{m}^{2}$ respectively. 


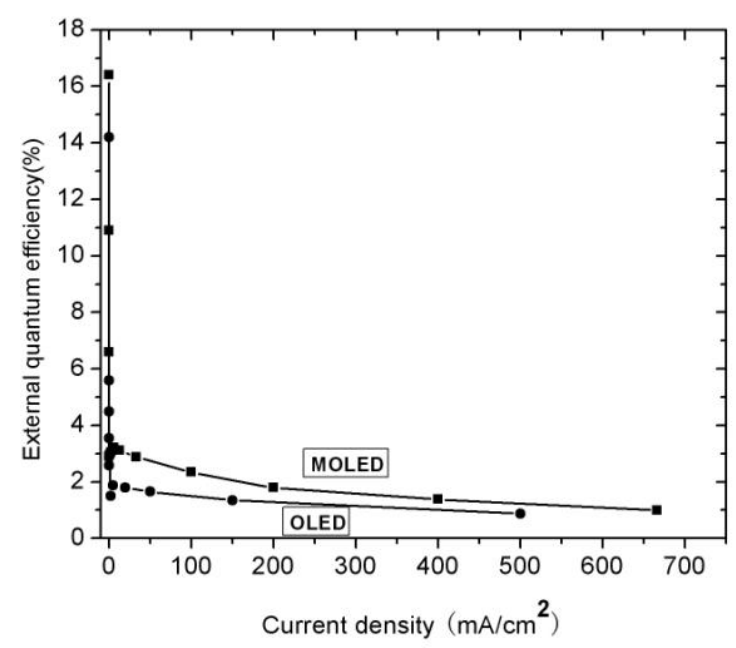

Fig.5 External quantum efficiency -current density characteristics of the MOLED and OLED

Fig.5 shows the external quantum efficiency-current density characteristics of the MOLED and OLED. It is clearly shown that the external quantum efficiency is decreased with the increase of current density. The maximum of MOLED is $16.4 \%$ and that of OLED is $14.2 \%$. But when the current density is the same, the external quantum efficiency of the MOLED is higher than the OLED, which is shown that the promotion of the luminance with the cavity is gotten.

\section{Conclusion}

Design and manufacture of two devices, microcavity blue phosphorescent organic electroluminescent devices (MOLED) and non-cavity device that is a blue phosphorescent organic electroluminescent device (OLED). Research on the impact of microcavity structure of organic electroluminescent devices emitting properties of blue phosphorescence. The results show that: the microcavity structure can improve the luminous performance of OLED. For MOLED, the maximum brightness can reach $9720 \mathrm{~cd} / \mathrm{m}^{2}$. The maximum external quantum efficiency is $16.4 \%$, the luminescence properties MOLED are better than OLED.

\section{References}

[1] Xiao Y, Yang J P,Cheng P P ,et al. Surface plasmon-enhanced electro luminescence in organic light-emitting diodes incorporating Au nanoparticles. Appl. Phys. Lett ,100(2012) : 013308-1-3.

[2] Yu Xin-dong, Wu Zhen-ying. W-OLED Lighting Technology Research. Advaanced Display,142(2012), 5-7.

[3] M Pope, H. Kallmann, P. Magnante, Electroluminescence in organic crystals J. Chem .Phys, 38(1963):2042-2043.

[4] C W Tang, S A Van Slyke. Organic electroluminescent diodes. Appl.Phys. Lett.,51(1987):913-915.

[5] Baldo M A, Brien D F, You Y,et al. Highly efficient phosphorescent emission from organic electroluminescent devices. Nature. 395(1998) : 151-154.

[6] Li Hongyan,Zhang Yuxiang,Zhang Hongke,et al. Effect of a series of host material on optoelectronic performance of red phosphorescent OLED .Chin. J. Lumin. 30(2009) : 585-589. 
[7] Yang Hui-shan. Fabrications of White Organic Light-Emitting Diode Based on Fluorescent Sub-Monolayer Combine with Phosphorescent Doping Layer. Acta Optica Sinica, 33(2013):0323005(1-6).

[8] Boo Young Jung, Nam Young Kim, Changhee Lee. Control of resonant wavelength from organic light-emitting materials by use of a Fabry-Perot microcavity structure [J]. Appl. Opt. 2002, 41(16): 3312-3318.

[9] J.-W. Shin, D.-H. Cho, J. Moon, et al., Random nanostructure scattering layer for suppression of microcavity effect and light extraction in OLEDs. Optics Letters, 39(2014):3527-3530.

[10] Zhang Chun-yu, Wang Cheng,Lu Jing-bing,et al. Spectral analysis of Organic/Microcavity organic light-emitting devices with the change of thickness of the organic layer[J]. Spectroscopy and Spectral Analysis, 2012,32(1) : 69-73. 